\title{
A Hybrid Approach of Facial Emotion Detection using Genetic Algorithm along with Artificial Neural Network
}

\author{
Amrendra Sharan \\ M.Tech (IT) \\ CDAC, Mohali
}

\author{
Sunil Kumar Chhillar \\ Principal Engineer \\ CDAC, Mohali
}

\begin{abstract}
The facial emotion recognition from facial expression is one of the most imperative intellectual functions that our brain performs quite efficiently. For a single person, facial expressions may be different at different instances and this is a great task to recognize the emotion from their facial expressions. This work is an attempt to look at the task of emotion recognition using artificial intelligence which is cognitively very attractive and the same has been shown to perform very well for emotion recognition. The facial emotion recognition is frequently used but there is a problem occurred during the classification of emotion from the facial expressions due to existing feature extraction techniques and their uniqueness. The major causes of the problem in facial emotion recognition system are the extraction of best and appropriate feature sets from the faces according to the facial expressions. To minimize these types of problems from facial emotion recognition system, SIFT descriptor along with genetic algorithm (GA) is best solution according to the survey and to achieve better performance of proposed work, a novel objective function is being designed. In the proposed work, artificial Neural Network (ANN) is used as a classifier to train the facial emotion recognition system and by using the public database Japanese Female Facial Expression (JAFFE), the accuracy of facial emotion recognition is obtained around $98 \%$ in MATLAB.
\end{abstract}

\section{Keywords}

Facial emotion recognition, Scale invariant feature Transform (SIFT), Artificial neural network (ANN), Genetic algorithm (GA)

\section{INTRODUCTION}

Emotion Recognition, these days is gaining an importance for its rising scope of applications in the system of human computer interaction. Emotion recognition is the process to recognize the human emotion in terms of voice, gesture, facial expression and posture [1]. According to the psychological theory, human emotions can be classified into six emotions comprises of surprise, fear, disgust, anger, happiness and sadness. When the emotions of the human changed, the muscles of the face as well as the tone of speech changed. These emotion signals can be recognized by human being, by processing the available information obtained by ears and eyes. In this paper, a facial emotion recognition system has been developed that recognizes the six emotions named as surprise, fear, disgust, anger, happiness and sadness [2].

\subsection{Facial Emotion Recognition}

Facial expression is a fundamental manner for communicating emotions of human beings, recognizes its applications in health care, HCI (Human-computer interaction), surveillance, driver safety, educational software, animations and computer games, emotion processing with deceit detection and so on. Figure 1 explains the face recognition system [3].

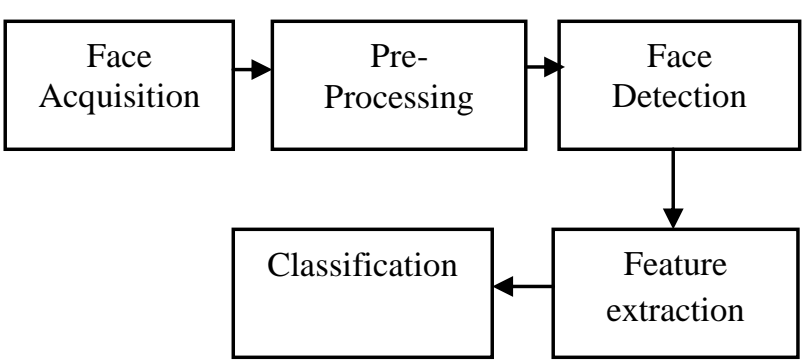

Fig 1: Facial Emotion Recognition System

The five stages used in the recognition of facial emotions are listed and defined below:

\subsubsection{Face acquisition}

The face is captured by using a sensor device like camera and then converted the captured image into digital form by using analog to digital convertor in case of analog camera.

\subsubsection{Pre-processing}

In pre-processing stage, the captured image is converted into gray scale and the size of the image also reduced. In this stage, only the irrelevant data is compressed and thus, enhances the important features of the image.

\subsubsection{Face detection}

After pre-processing, face of the captured image has been detected on the basis of threshold method, edge detection and region based methods.

\subsubsection{Feature extraction}

Feature extraction is technique to extract only the required features of the face i.e. it finds the key value points using SIFT.

\subsubsection{Classification}

This is the last stage used in the face emotion reorganization that depends upon the generated features using feature extraction technique. In this paper, ANN is used as a classifier to train the proposed facial emotion recognition system based on optimized feature and on the basis of training of system, the classification of a test image from training structure of ANN can be done [4].

\section{PROPOSED TECHNIQUES}

In this section, the techniques used for feature extraction, optimization and classification of the captured image have been discussed. For feature extraction, Scale Invariant Feature Transform (SIFT), for optimization Genetic algorithm (GA) and for classification purpose, Artificial neural network (ANN) have been used. 


\subsection{SIFT (Scale Invariant Feature Transform)}

To match features among different images is a common problem in computer vision. When the images with different scale and rotations are available Scale invariant feature transform feature extraction technique performs well [5]. It is an image descriptor used for image based matching with the recognition give by David Lowe. Different steps used by SIFT algorithm to work well are listed below:
i. Initially Scale invariance has been constructed by using Scale space.
ii. Interesting points also known as key points of the image are finding by using Laplacian of Gaussian approximation method.
iii. Maxima and minima points of the Gaussian image have been calculated.
iv. Edges and low contrast points of the tested image are eliminated by using some detector.
v. Orientation of each point is calculated.

Then features are identified by using SIFT $[6,7]$.

\subsection{GA (Genetic Algorithm)}

Genetic algorithm is used to solve the optimization problems on the basis of natural selection given by John Holland in 1970 's. GA constantly modifies the population of individual solutions. At every step, GA selects individuals randomly from the current population to be parents and utilizes them for producing the children for the subsequent generation. GA utilizes three major types of rules at every step for creating the next generation from the current population:
i. Selection, rules select the individuals, known as parents, which contribute to the population at the next generation.
ii. Crossover, rules integrates two parents to produce children for the next generation.
iii. Mutation, rules apply random changes to individual parents to have children $[8,9]$.

In fitness function of proposed work, Fs is the current selected key point and $\mathrm{Ft}$ is the threshold value of key points and it is the average value of SIFT key points. On the basis of given condition, genetic algorithm optimizes the feature set and create a unique feature sets using the optimal value.

$$
\mathrm{f}(\mathrm{fit})= \begin{cases}\text { True, } & \mathrm{fs} \geq \mathrm{ft} \\ \text { False, } & \mathrm{fs}<\mathrm{ft}\end{cases}
$$

Where $\mathrm{f}$ (fit) is fit key points according to the fitness function. Here, True and False represents the selection of key points value, if $\mathrm{Fs}$ is less than $\mathrm{Ft}$ then selected value is false and replace by the threshold value and store in optimized feature sets, if Fs doesn't satisfy the condition then selected feature value Fs is true and as it is store in optimized feature sets as optimized key points.

\subsection{ANN (Artificial Neural Network)}

ANN is a processing device consisted of interconnected large number of processing nodes that processes the input by their dynamic state response and produce output. Neural network mainly comprises of three layers named as input, hidden and output layer [10]. Layers consist of number of nodes that are interconnected to each other. Input layer is followed by one or more hidden layers. In the hidden layer, actual processing of the input data is done by using some weights attached to the nodes [11]. Figure 2 explains the architecture of architecture of artificial neural network.

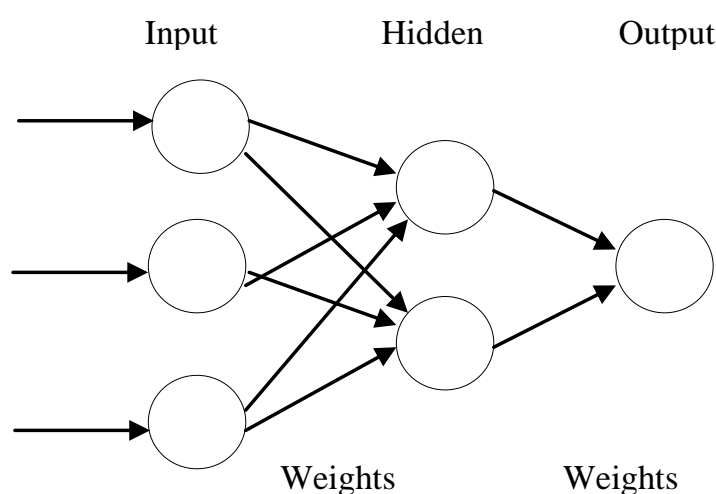

Fig 2: Artificial neural network

ANN contains some learning rules, which modified the weights according to the input information. The delta learning rule is used mostly that is also known as Back propagation neural network (BPNN) [12].

\section{RELATED WORK}

P. Lucey et al. [1] conducted two types of experiments named as AU detection and emotion detection being implemented on the $\mathrm{CK}+$ database. For Automatic facial action unit (AU) detection and emotion detection, 123 and 118 training and testing sets are considered. S. Wang et al. [2] proposed a facial expression database that contains both spontaneous and posed expressions captured by an analog camera. Face recognition is performed by using Principle component analysis (PCA) and Linear Discriminator Analysis (LDA) to identify the expressions from the camera. Mikhailova, Elena S., et al [3] recognized the facial expression of emotions in major depressive disorder of patient. A. C. Cruz et al. [4] proposed a principled technique for addressing the facial emotion expressions using down sampling method. Data base used for experimentation is Cohn-Kanade and MMI facial expression. For classification, SVM has been used as a classifier. H. Soyel and H. Demirel [5] presented a technique based on Scale Invariant Feature Transform (SIFT) used to increase the identification of facial expressions in different poses. SVD has been used as a classifier to recognize the true facial expression. Table 1 is defining the comparison of existing techniques on the basis of database used, proposed techniques, outcome and advantages.

Table 1. Comparison of existing techniques

\begin{tabular}{|c|c|c|c|c|}
\hline AUTHORS & DATABASE USED & PROPOSED \\
TECHNIQUES & OUTCOMES & ADVANTAGES \\
\hline $\begin{array}{c}\text { S. Yang and B. } \\
\text { Bhanu [6] }\end{array}$ & $\begin{array}{c}\text { GEMEP-FERA dataset, } \\
\text { used 155 videos to } \\
\text { trained data }\end{array}$ & $\begin{array}{c}\text { Local Binary } \\
\text { Pattern (LBP) and } \\
\text { Local phase } \\
\text { Quantization (LPQ) } \\
\text { SIFT, SVM }\end{array}$ & $\begin{array}{c}\text { Average rate has been } \\
\text { measured for five emotions } \\
\text { include anger, fear, joy, relief } \\
\text { and sadness. }\end{array}$ & $\begin{array}{c}\text { Perform well on unseen } \\
\text { data. }\end{array}$ \\
\hline
\end{tabular}




\begin{tabular}{|c|c|c|c|c|}
\hline $\begin{array}{c}\text { Berretti, S et al. } \\
\text { [7] }\end{array}$ & BU-3DFE database & SIFT and SVM & $\begin{array}{l}\text { Recognition rate has been } \\
\text { measured which is about } 78.43 \\
\% \text { for six facial emotion. }\end{array}$ & $\begin{array}{l}\text { Used for recognizing 3D } \\
\text { facial expression images. }\end{array}$ \\
\hline $\begin{array}{l}\text { G. G. Yen and N. } \\
\text { Nithianandan [8] }\end{array}$ & $\begin{array}{l}\text { Kodak Digital Science } \\
\text { DC260 zoom camera } \\
\text { with a resolution of } 1024 \\
\text { by } 1536 \text { pixels. } \\
\end{array}$ & Genetic algorithm & $\begin{array}{l}\text { Face with different facial } \\
\text { expressions has been identified. }\end{array}$ & $\begin{array}{l}\text { Decrease computation } \\
\text { complexity and hence } \\
\text { reduce search time }\end{array}$ \\
\hline $\begin{array}{c}\text { Goyal, Rashi, and } \\
\text { Tanushri Mittal } \\
{[10]}\end{array}$ & $\begin{array}{l}\text { Self-made database is } \\
\text { used by capturing the } \\
\text { face images using front } \\
\text { cameras and videos of } \\
\text { about } 50 \text { people's } \\
\text { emotions. }\end{array}$ & $\begin{array}{l}\text { Artificial neural } \\
\text { network }\end{array}$ & $\begin{array}{l}\text { Confusion matrix } \\
\text { regression plot have been } \\
\text { calculated to know the } \\
\text { performance of the system. }\end{array}$ & $\begin{array}{l}\text { Detect the objects of } \\
\text { various sizes. }\end{array}$ \\
\hline Neelam et al. [11] & $\begin{array}{l}\text { Jaffe and Paul Ekman } \\
\text { database }\end{array}$ & $\begin{array}{l}\text { Neural network, } \\
\text { PCA }\end{array}$ & $\begin{array}{l}\text { Accuracy rate up to } 76 \% \text { has } \\
\text { been obtained for four type of } \\
\text { facial expressions named as } \\
\text { happy, sad, angry and surprised }\end{array}$ & \\
\hline $\begin{array}{c}\text { Ioannou, Spiros V } \\
\text { et al. [12] }\end{array}$ & naturalistic database & Neuro- fuzzy & $\begin{array}{l}\text { The recognition rate up to } 58 \% \\
\text { has been obtained. }\end{array}$ & Classification rate is high \\
\hline
\end{tabular}

\section{SIMULATION ENVIRONMENT}

The simulation of the proposed work has been carried out in MATLAB environment using Core 2 Duo or higher CPU having memory size of $3 \mathrm{MB}$ in window 7 platform. The database used in the proposed work is JAFFE that contains six types of facial expressions.

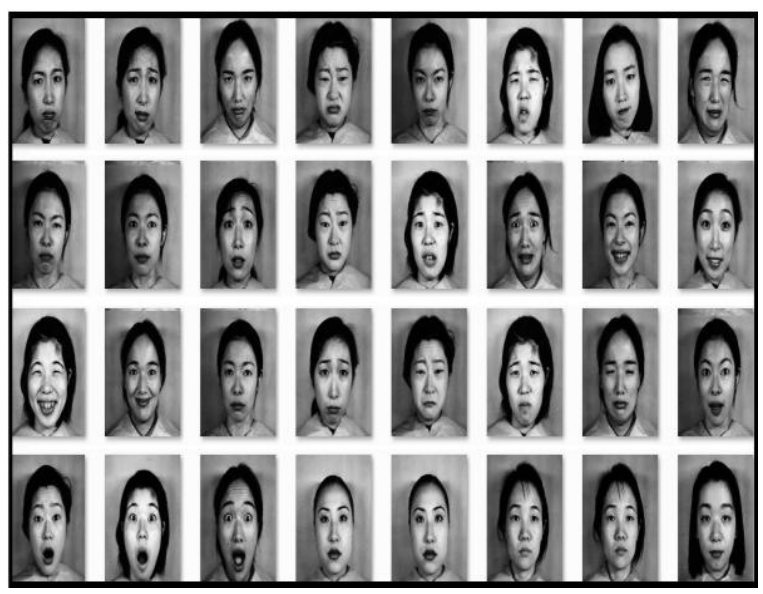

Fig 3: JAFFE Database

Above figure 3 represents the database used in the proposed Facial Emotion Detection using Artificial Neural Network. The database contains mainly 6 types of facial expressions with neutral posed by Japanese female models. The format of images in the database is TIFF without any type of compression. The facial expressions present in the database are Angry, Disgusted, Fear, Happy, Sad and Surprised. The facial expressions present in the database are given below in figure 4.

Below figure 4 represents different types of facial expressions which are used in the database of the proposed facial emotion detection work. As shown above, figure 4(a) is the angry facial expressions, 4(b) is the disgusted facial expressions, 4(c) is the fear facial expressions, 4(d) is the happy facial expressions, and 4(e) is the sad facial expressions and 4(f) is the surprised facial expressions.

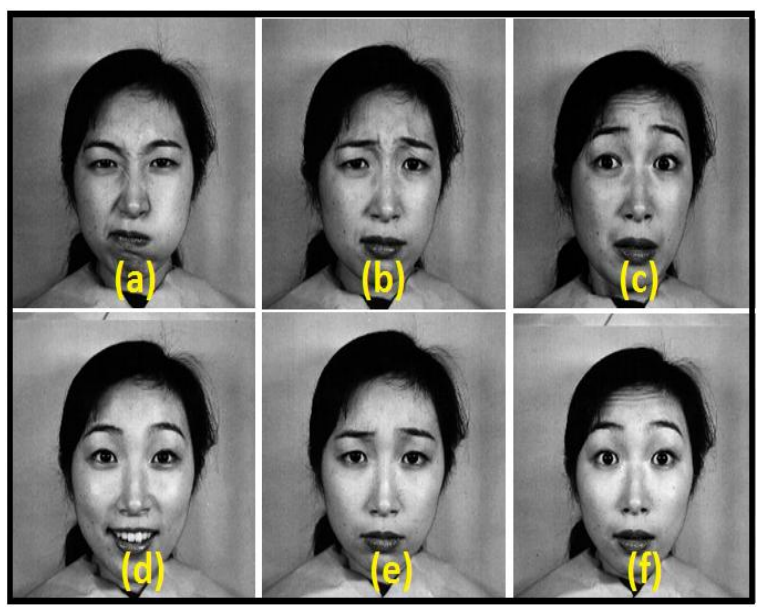

Fig. 4 Sample images in Database with different types of emotions (a) Angry, (b) Disgusted, (c) Fear, (d) Happy, (e) Sad and (f) Surprised

\section{EXPERIMENTAL RESULTS}

In this section, the facial emotion of a test image along with the methodology used for recognizing the facial emotion of the test image are described.

\subsection{Methodology}

To verify the efficiency and accuracy of proposed facial emotion recognition system using SIFT descriptor along with genetic algorithm and the artificial neural network as a classifier; several experiments with this procedure on several images are performed. In the proposed facial emotion recognition system, there are several steps used for recognizing the emotions from the face images. The methodology of proposed work is given below and is shown in figure 5.

Step 1: A proper GUI for the proposed facial emotion recognition system is designed and developed.

Step 2: Face images with different facial expression for Training and Testing of proposed facial emotion recognition system are uploaded.

Step 3: Pre-processing on uploaded face images in both section is applied. 
Step 4: A code for the face detection from the pre-processed face images in training as well as testing section is developed.

Step 5: SIFT Descriptor for the feature extraction from the detected face is applied.

Step 6: Genetic Algorithm to optimize SIFT features and remove the unwanted feature sets using the novel objective function is initialized.

Step 7: Artificial Neural Network is applied on optimized data to train the database and to train the data using following steps:

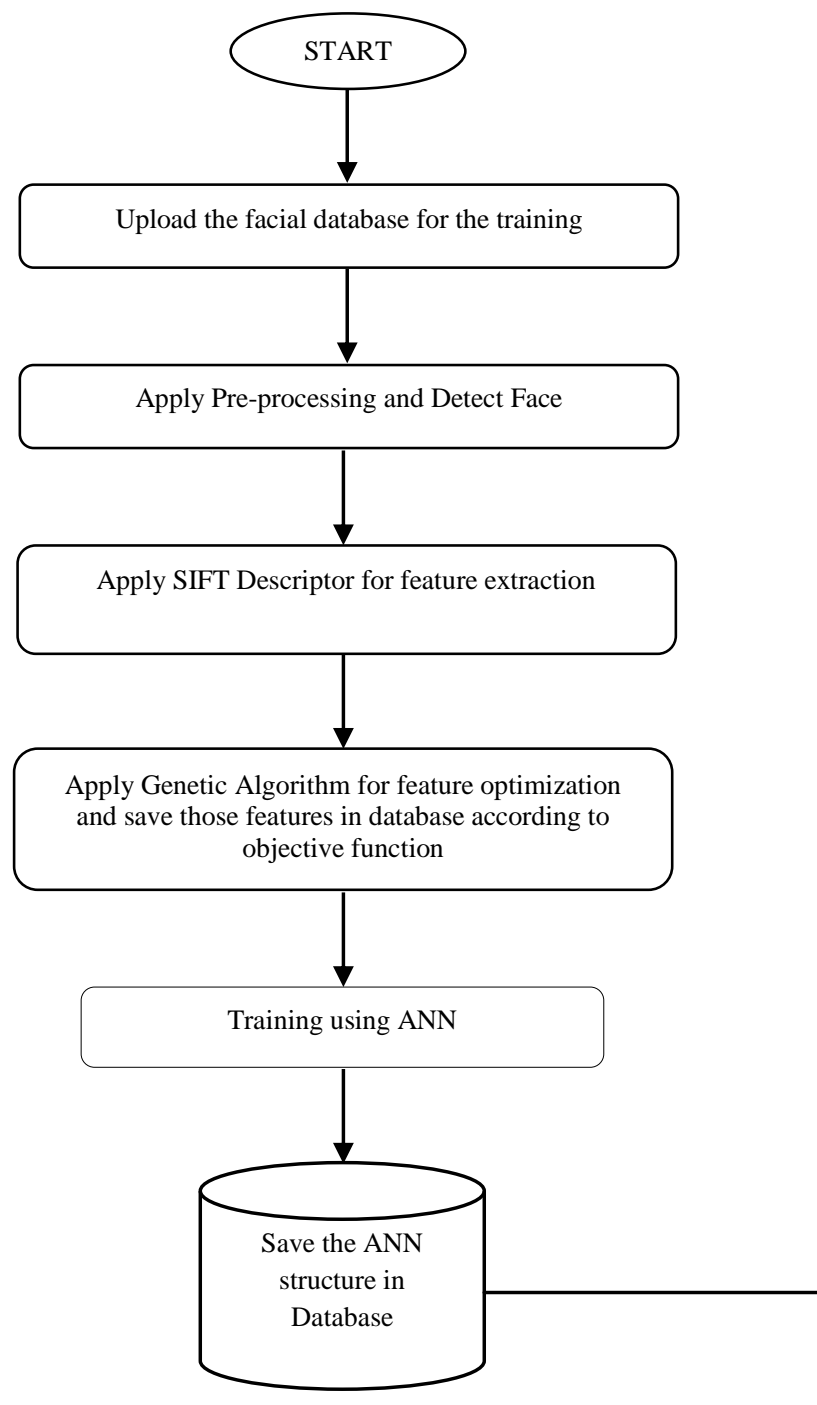

Optimized feature as an input of artificial neural network for training and testing data are selected.

Total categories which are generated by the training of optimized data using artificial neural network are categorized.

Step 8: After that, in the classification section, the test data according to the trained artificial neural network structure is classified.

Step 9: At last of the module, the performance parameters of proposed facial emotion recognition system like FAR, FRR and Accuracy are calculated.

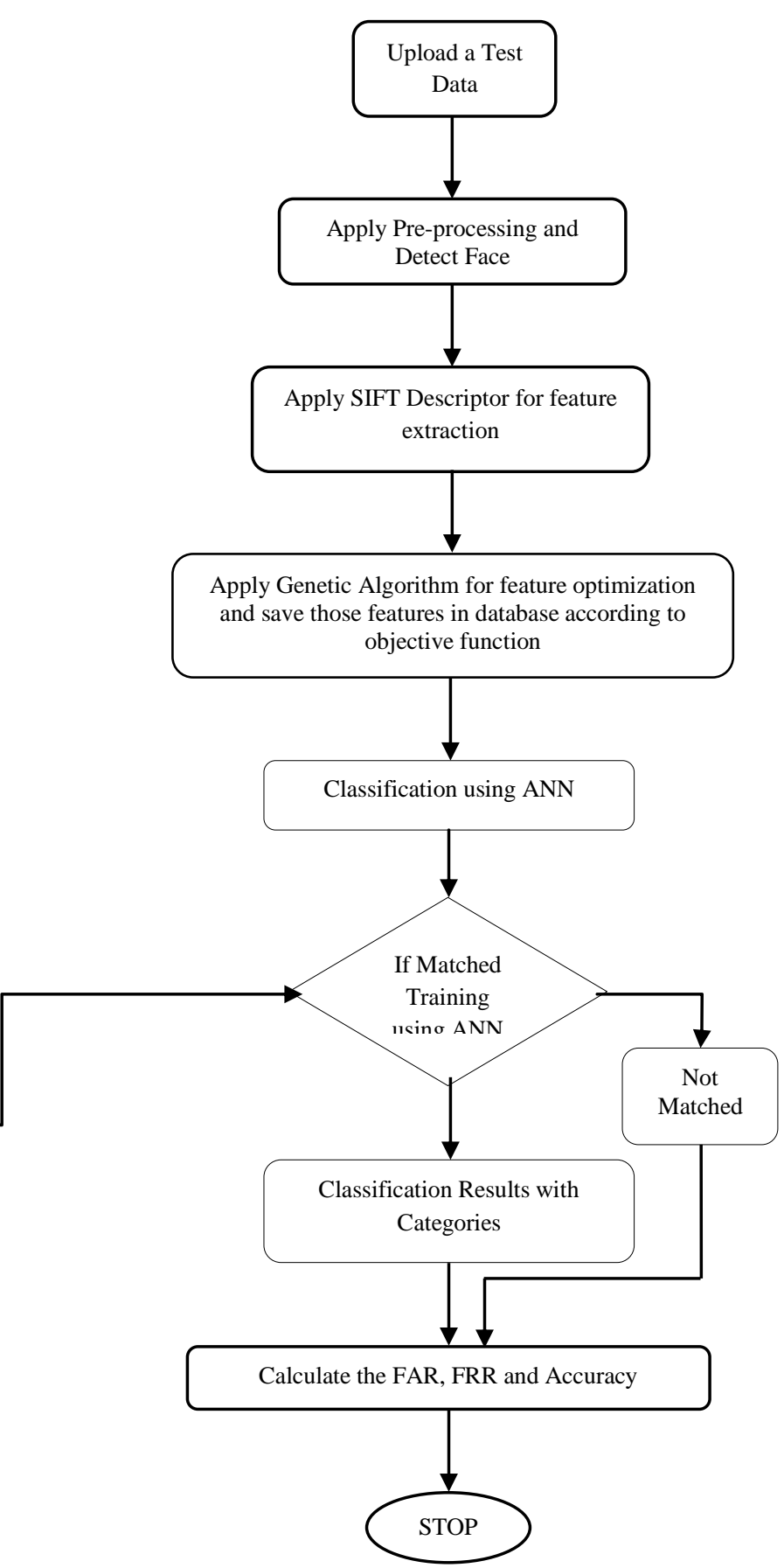

Fig. 5 Proposed Methodology Flowchart 


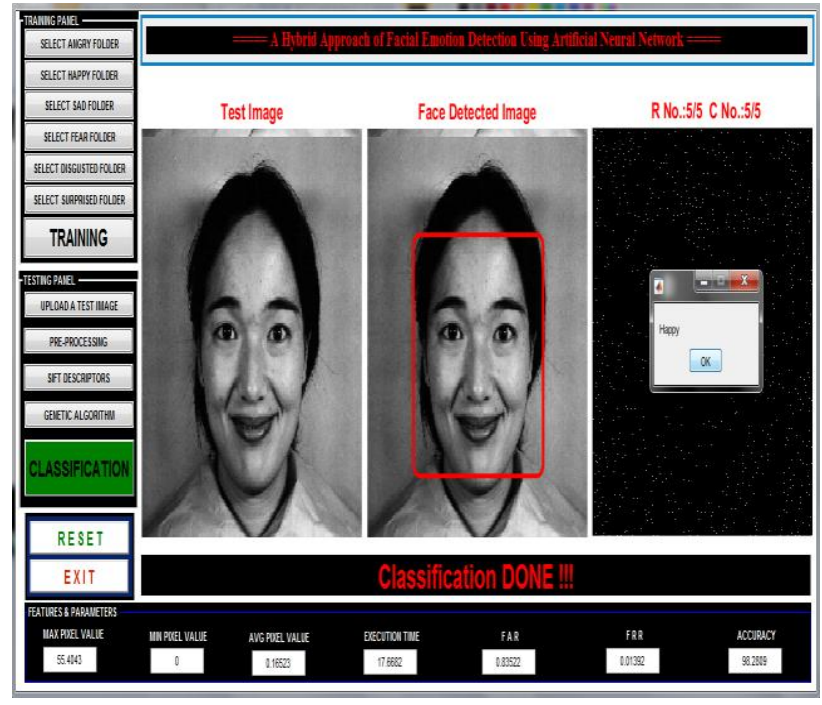

Fig. 6 Classification of emotion recognition system

Test image uploaded by clicking on uploaded test image button is shown in figure 6. Pre-processing is applied to convert the color image into gray image and resize the image. Feature extraction technique that is SIFT is applied on the tested image to find the key points of the detected image. After that, the optimization is done using Genetic algorithm to optimize the extracted features and ANN is used to classify the optimized features. The parameters calculated are listed below in table 3 :

Table 3: Comparison of accuracy with proposed and existing work

\begin{tabular}{|c|c|}
\hline \multicolumn{2}{|c|}{ Accuracy (\%) } \\
\hline $\begin{array}{c}\text { Existing work (Log Gabor } \\
\text { filter) }\end{array}$ & Proposed work (SIFT) \\
\hline 88.8 & 98.27 \\
\hline
\end{tabular}

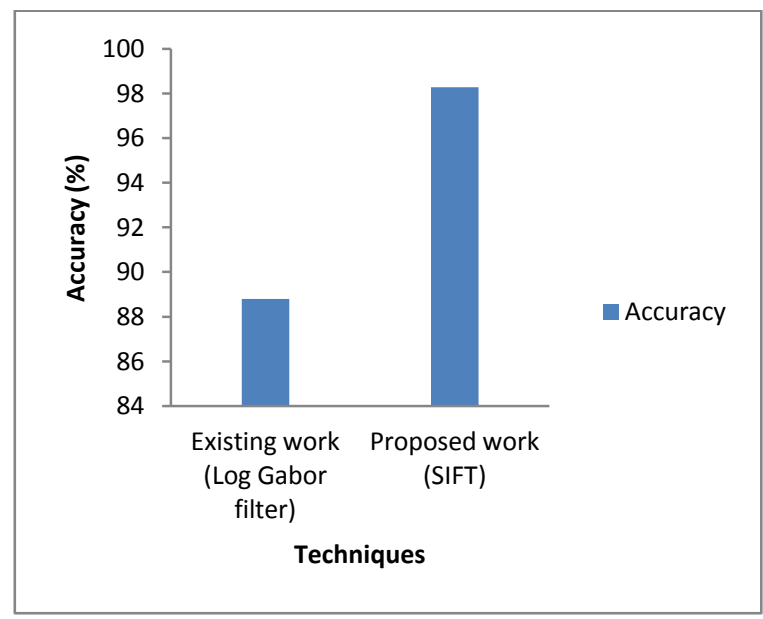

Fig. 8 Comparison of accuracy with proposed and existing work

Above figure 8 displays the comparison of accuracy w.r $t$ existing techniques. For Log Gabor filter, the accuracy obtained is $88.8 \%$ whereas for the proposed work, average accuracy has been increased and came out to be $98.27 \%$.
The comparison of FAR, FRR and Accuracy is shown in table 4.

Table 4: FAR, FRR and accuracy for the proposed work

\begin{tabular}{|c|c|c|c|}
\hline S. no. & FAR & FRR & Accuracy \\
\hline 1 & 0.83522 & 0.01392 & 98.3809 \\
\hline 2 & 0.83769 & 0.01547 & 97.4872 \\
\hline 3 & 0.82357 & 0.02354 & 96.2478 \\
\hline 4 & 0.83467 & 0.03457 & 97.2547 \\
\hline 5 & 0.82475 & 0.07589 & 98.25476 \\
\hline 6 & 0.81752 & 0.04758 & 99.1275 \\
\hline 7 & 0.81457 & 0.24783 & 98.2457 \\
\hline 8 & 0.81245 & 0.01457 & 97.0214 \\
\hline 9 & 0.83213 & 0.07821 & 99.0547 \\
\hline 10 & 0.79257 & 0.05471 & 98.5749 \\
\hline
\end{tabular}

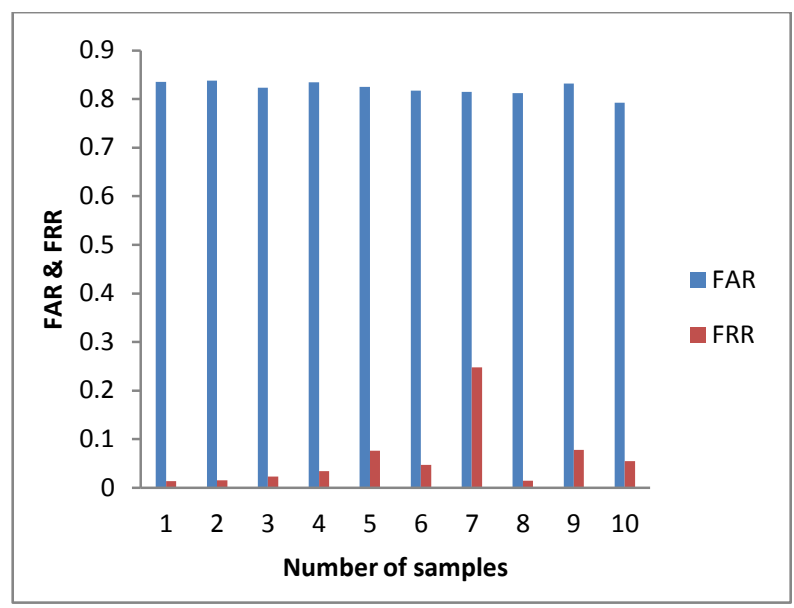

Fig. 9 FAR and FRR of the proposed work

As shown in figure 9, blue bar line indicates the FAR values obtained for 10 numbers of samples and red bar line indicates the FRR values obtained for the proposed work.

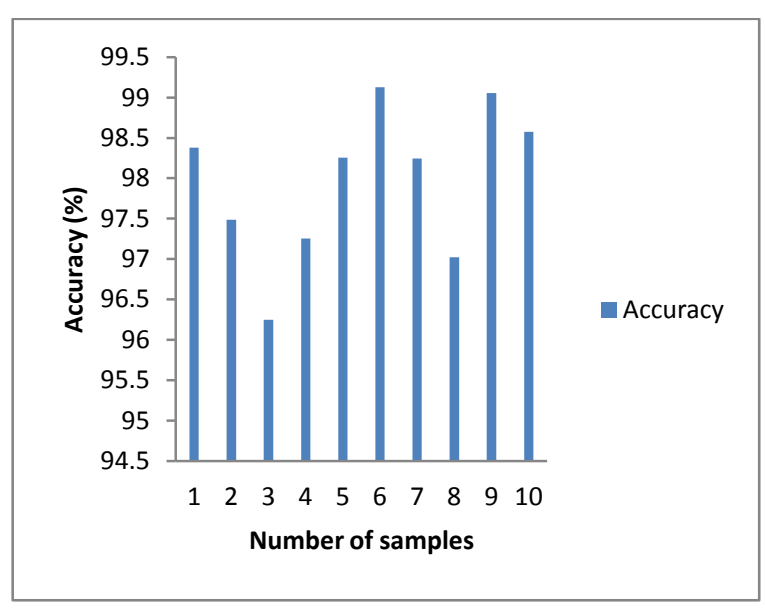

Fig. 10 Accuracy of the proposed work

In the above figure $10, \mathrm{X}$-axis represents the number of samples and $y$-axis represents the achieved accuracy of the proposed work in percentage. The accuracy of the proposed work varies from 96.2478 to 99.0547 . From the above figure, the average accuracy of the proposed work is more than $98 \%$. 


\section{CONCLUSION}

In this research work, a hybrid approach of facial emotion detection using genetic algorithm along with artificial neural network has been proposed. In facial emotion recognition system, different emotions like surprise, fear, disgust, anger, happiness and sadness are recognized from facial expressions. To recognize an emotion from facial expression, feature extraction plays an important role to achieve a better accuracy in minimum execution time. In the proposed work, SIFT feature descriptor has been used to extract the features from different types of facial expressions and to create an optimized feature set, genetic algorithm has been used with novel fitness function. Artificial neural network has been used as a classifier to train proposed emotion recognition system with different type of emotions images. Japanese Female Facial Expression (JAFFE) dataset has been used to simulate the proposed work in MATLAB environment. The accuracy of proposed facial emotion recognition system is more than 98 $\%$ with an acceptable execution time.

In future work, as a feature extraction technique, region based feature extraction techniques like BRISK; MSER can be used along with artificial neural network (ANN). For optimization, more than one optimization can be used in hybrid form.

\section{REFERENCES}

[1] Lucey, P., Cohn, J.F., Kanade, T., Saragih, J., Ambadar, Z. and Matthews, I., 2010. The extended cohn-kanade dataset (ck+): A complete dataset for action unit and emotion-specified expression. In Computer Vision and Pattern Recognition Workshops (CVPRW). IEEE Computer Society Conference on IEEE, 94-101.

[2] Wang, Shangfei, Zhilei Liu, Siliang Lv, Yanpeng Lv, Guobing Wu, Peng Peng, Fei Chen, and Xufa Wang. 2010. A Natural Visible and Infrared Facial Expression Database for Expression Recognition and Emotion Inference. IEEE Transactions on Multimedia. 12. 7, 682691.

[3] Mikhailova, E. S., Vladimirova, T. V., Iznak, A. F., Tsusulkovskaya, E. J., \& Sushko, N. V.1996. Abnormal recognition of facial expression of emotions in depressed patients with major depression disorder and schizotypal personality disorder. Biological psychiatry. 40, 697-705.

[4] A.C. Cruz, B. Bhanu and N. S. Thakoor. 2014. Vision and Attention Theory Based Sampling for Continuous Facial Emotion Recognition. IEEE Transactions on Affective Computing. 5. 4, 418-431.

[5] H. Soyel and H. Demirel.2011. Improved SIFT matching for pose robust facial expression recognition. Face and Gesture. Santa Barbara, CA, 585-590.

[6] S. Yang and B. Bhanu.2011.Facial expression recognition using emotion avatar image. Face and Gesture, Santa Barbara, CA, 866-871.

[7] Berretti, S., Amor, B. B., Daoudi, M., \& Del Bimbo, A. 2011. 3D facial expression recognition using SIFT descriptors of automatically detected keypoints. The Visual Computer, 1021.

[8] G. G. Yen and N. Nithianandan. 2002. Facial feature extraction using genetic algorithm. Evolutionary Computation. Honolulu. HI, 1895-1900.

[9] Elsayed, Saber M., Ruhul A. Sarker, and Daryl L. Essam. 2014. A new genetic algorithm for solving optimization problems. Engineering Applications of Artificial Intelligence. 27, 57-69.

[10] Goyal,Rashi, and Tanushri Mittal. Facial Expression Recognition using Artificial Neural.

[11] Neelam, Manisha Dr Jagjit Singh Dr, and R. Prakash.2014. Facial Expression Recognition Using Neural Network. International Journal of Technology Innovations and Research. 10, 2321-1814.

[12] Ioannou, S. V., Raouzaiou, A. T., Tzouvaras, V. A., Mailis, T. P., Karpouzis, K. C., \& Kollias, S. D, 2005. Emotion recognition through facial expression analysis based on a neurofuzzy network. Neural Network.s18, 423-435. 